\title{
The effect of a Computerised Decision Support System (CDSS) on compliance with the prehospital assessment process: results of an interrupted time-series study
}

\author{
Magnus Andersson Hagiwara ${ }^{1,2 *}$, Björn-Ove Suserud ${ }^{1}$, Boel Andersson-Gäre², Bengt- Arne Sjöqvist ${ }^{3}$,
} Maria Henricson ${ }^{2}$ and Anders Jonsson ${ }^{1}$

\begin{abstract}
Background: Errors in the decision-making process are probably the main threat to patient safety in the prehospital setting. The reason can be the change of focus in prehospital care from the traditional "scoop and run" practice to a more complex assessment and this new focus imposes real demands on clinical judgment. The use of Clinical Guidelines (CG) is a common strategy for cognitively supporting the prehospital providers. However, there are studies that suggest that the compliance with CG in some cases is low in the prehospital setting. One possible way to increase compliance with guidelines could be to introduce guidelines in a Computerized Decision Support System (CDSS). There is limited evidence relating to the effect of CDSS in a prehospital setting. The present study aimed to evaluate the effect of CDSS on compliance with the basic assessment process described in the prehospital CG and the effect of On Scene Time (OST).
\end{abstract}

Methods: In this time-series study, data from prehospital medical records were collected on a weekly basis during the study period. Medical records were rated with the guidance of a rating protocol and data on OST were collected. The difference between baseline and the intervention period was assessed by a segmented regression.

Results: In this study, 371 patients were included. Compliance with the assessment process described in the prehospital CG was stable during the baseline period. Following the introduction of the CDSS, compliance rose significantly. The post-intervention slope was stable. The CDSS had no significant effect on OST.

Conclusions: The use of CDSS in prehospital care has the ability to increase compliance with the assessment process of patients with a medical emergency. This study was unable to demonstrate any effects of OST.

\section{Background}

Prehospital emergency care is characterized by judgement and decision making (JDM) in an unstable setting [1]. Prehospital clinicians have had to make advanced medical decisions a long distance from medical support, they have to assess and treat patients with many different symptoms and conditions in altered settings. The typical ambulance mission can be divided in 7 phases; receiving the call, arriving at the address, performing an on-scene

\footnotetext{
* Correspondence: magnus.hagiwara@hb.se

${ }^{1}$ University of Borås, School of Health Sciences, 50190 Borås, Sweden

${ }^{2}$ School of Health Sciences, Jönköping University, Jönköping 551 11, Sweden Full list of author information is available at the end of the article
}

assessment, performing an initial patient assessment, transport the patient to the ambulance, performing additional assessment and treatment on route, arriving to the hospital and handing over the patient [1]. Research has suggested that it is in the on-scene phase of an ambulance mission that imposes the greatest demands on the prehospital clinicians JDM process. It is outside the ambulance the clinicians have access to limited cognitive support and have to deal with varying settings [2]. In a case study with the aim to investigate the use of guidelines and protocols among prehospital clinicians in Sweden [3], the greatest obstacle to the use of guidelines was their physical format, which was due to the development 
process, which produces guidelines that are not suited to use in the prehospital setting. The consequence of the poor format were that the guidelines were seldom used explicit in the on scene patient assessment [3].

The limited research in prehospital patient safety suggests that most important issues related to patient safety is errors in the JDM process among prehospital clinicians [4]. Except the unstable settings were the prehospital care is execute, there can be several reasons to poor JDM processes. One suggestion is that prehospital clinicians not always are supported with right tools or that the education is not adequate for the complex work [5]. Other reasons could be lack of feedback [6], fatigue, stress [7], motivation and morale [8]. The use of cognitive aids such as guidelines, protocols, checklists and algorithms is considered as important in the execution of safe prehospital care [9]. However, there are studies that suggest that compliance with guidelines in some cases is low in the prehospital setting [10-14]. There can be several reasons for poor compliance with guidelines. The implementation strategies are one possible cause [15], while factors such as guideline visibility, content, design and the level of evidence have been shown in other health-care settings to have an impact on compliance with guidelines [16].

In in-hospital emergency settings, computerised decision support have been proved to have the ability to increase compliance to guidelines and processes of care [17]. Four features have been identified as important factors for the ability of decision support systems to improve clinical practice; the decision support is computer based, gives support as part of natural workflow, the decision support is delivered at the time and location of the decision making and actionable recommendations are provided [18]. There is limited evidence relating to the effect of CDSS in a prehospital setting [19]. In a recent simulation study of a CDSS effect of compliance to guidelines and CDSS effect of on-scene time (OST), improved guideline compliance was found among the ambulance clinicians who used a CDSS in two simulated patient cases compared with those that used guidelines in the usual paper format, but the group using the CDSS also spend more time on-scene [19].

Most studies of guideline adherence in prehospital care have studied adherence to single diagnosis and treatment plans. Then bias in the JDM process seems to be the major threat against patient safety in prehospital care; we aimed to evaluate the CDSS effect on the basic assessment process of the patient with medical emergencies.

A poorly designed CDSS can potentially produce harm. For examples a poorly designed interface that are unclear or irrational can results in error even among computer experience users [20]. A poorly design CDSS in prehospital care could results in an increased on-scene time (OST). In some emergencies, the time spent on-scene can negatively affect patient outcome [21]. The second aim was therefore to measure the OST time, defined as the time when the ambulance arrives at the address to the time when transportation is initiated.

\section{Methods}

\section{Setting and participants}

The study was conducted at a single ambulance station in south-west Sweden. The station's catchment area covers both rural and urban areas. Most of the patients are transported to the nearest hospital, which is located 37 kilometers from the ambulance station. The ambulance undertook approximately 1900 missions in 2012. The entire regular employed at the station participated in the study. The regular employed comprises nine ambulance nurses (AN) and one emergency technician (EMT). Of them six person are male and four female. The average age is 45.9 years (range 34-60) and the average experience from prehospital care is 16.9 years (range 2-35). Patients with symptoms of the most common medical emergencies described in the local prehospital guidelines were eligible for the study. This included patients with symptoms of chest discomfort, breathing problems, neurological symptoms, allergic symptoms, abdominal pain, affected circulation, including failing heart-conducting system, affected general condition, infections and endocrine system symptoms.

The exclusion criteria were:

- Patients $<18$ years.

- Patients suffering from trauma or poison.

- Patients transported between hospitals.

- Patients who were pregnant.

- Patients with no circulation and breathing.

The exclusion of patients who were pregnant or had some kind of trauma was due to the fact that, at this stage, the CDSS did not deal with these problems. A demographic description of the patients who were included can be found in Table 1.

A more detailed context description can be found in a previous study [3].

\section{Intervention}

The evaluated intervention was a Computerized Decision Support System (CDSS) designed for prehospital care. The CDSS can be defined as an expert system [22] and guides the prehospital clinicians through a systematic assessment process based on the content of the Advanced Medical Life Support (AMLS) system [23]. The CDSS was handheld (Panasonic Toughbook) and used the MobiMed 4.0 pre-hospital eHealth platform from Ortivus AB. The prehospital clinicians were trained to use the CDSS when 
Table 1 Study objects characteristics and disposition

\begin{tabular}{|c|c|c|c|}
\hline Variable & Pre-Intervention ( $\mathrm{n}=175$ ) & Post-Intervention ( $n=196$ ) & p-value \\
\hline Age, years, mean (SD) & $66.7(19.9)$ & $69.7(20.5)$ & 0.04 \\
\hline Sex, male (\%) & 43.4 & 54.1 & 0.04 \\
\hline Sex, female (\%) & 56.6 & 45.9 & \\
\hline \multicolumn{4}{|l|}{ Diagnostic category } \\
\hline Cardiovascular symptoms (\%) & 19.4 & 21.9 & 0.86 \\
\hline Respiratory symptoms (\%) & 17.7 & 14.3 & \\
\hline Neurological symptoms (\%) & 28.0 & 29.6 & \\
\hline Gastrointestinal symptoms (\%) & 15.4 & 15.8 & \\
\hline Effected general conditions (\%) & 2.3 & 1.5 & \\
\hline Effected circulation including failing heart conducting system (\%) & 9.1 & 7.1 & \\
\hline Infections (\%) & 4.6 & 6.6 & \\
\hline Endocrine system symptoms (\%) & 1.7 & 2.0 & \\
\hline Allergic symptoms (\%) & 1.7 & 0.5 & \\
\hline \multicolumn{4}{|l|}{ Number of patient assessed per ambulance personnel } \\
\hline Ambulance personnel 1 & 17 & 24 & 0.29 \\
\hline Ambulance personnel 2 & 20 & 14 & \\
\hline Ambulance personnel 3 & 15 & 16 & \\
\hline Ambulance personnel 4 & 16 & 27 & \\
\hline Ambulance personnel 5 & 26 & 23 & \\
\hline Ambulance personnel 6 & 17 & 23 & \\
\hline Ambulance personnel 7 & 16 & 19 & \\
\hline Ambulance personnel 8 & 18 & 19 & \\
\hline Ambulance personnel 9 & 13 & 11 & \\
\hline Ambulance personnel 10 & 17 & 10 & \\
\hline
\end{tabular}

The significance of continues variables was based on independent T-test and categorical variables Chi-square tests.

they performed the patient assessment. The CDSS consists of four main areas.

\section{First survey}

The goal of the first survey is to assess and treat lifethreatening conditions. It starts with an assessment of the airways and continues with an assessment of breathing, circulation and disability. During the assessment, the ambulance clinicians can also choose to receive additional support when it comes to assessing and treating problems in the different areas by using optional algorithms. For example, when treating a difficult airway, the CDSS provides an optional algorithm over airway management in 5 steps.

\section{History}

When the first survey is finished, the CDSS continues with focused history collection.

The CDSS guides the prehospital clinicians through a battery of questions. The questions start with signs and symptoms and continue with questions relating to onset, palliation, quality, radiation, severity, time, allergies, medication, past medical history, last oral intake and events prior to illness.

\section{Chief symptoms}

After finishing the history collection, the prehospital clinicians have to choose the patient's chief symptom. Examples of symptoms are chest discomfort, breathing problems, abdominal pain and altered mental status. When a chief symptom has been chosen, the CDSS provides a list of further focused medical assessments based on the symptom.

\section{Field diagnosis}

The last page in the CDSS is field diagnosis. Here, the CDSS provides a list of field diagnoses based on the chief symptom. Every field diagnosis is linked to the local prehospital guidelines where the ambulance staff can obtain information about diagnoses and also obtain access to treatment plans. The CDSS is also linked to a medical record system. All the actions performed in the CDSS are documented in the system. The documentation 
comprises ticking boxes and free text fields. When the patient assessment is complete, a medical record can be printed out for use in the hand-over phase.

Unfortunately, we were not able to configure the CDSS with the ordinary prehospital records system. The study participants had to make the first documentation in the CDSS and, later at the hospital, new documentation was created on a stationary computer in the Emergency Room (ER).

Apart from using the CDSS in the assessment of the patient, the participants were instructed to work as usual.

\section{Study design and outcome measures}

The study had a two-phase time-series intervention design [24], where study data were collected on a weekly basis during the study period. The Quality Criteria for Interrupted Time Series (ITS) Designs [25] checklist was used as a guide.

The study was conducted from November 2012 to May 2013. November, December, January made up the baseline period, when the clinicians worked as usual, supported by paper-based guidelines. The usual guidelines are conducted of three different systems; 1) the main guideline which contains description of assessment of the medical and trauma patient. It also contains description of different symptoms and conditions, directions for treatments and pathway protocols. The main guideline is a file with 194 A4 pages and is located between the seats in the front of the ambulance and one copy in the back of the ambulance. 2) A pocket guideline containing tables of drug doses, normal values and a few algorithms. The ambulance clinicians usually have the pocket guideline in a leg pocket. 3) The triage protocol which is a triage guide in a text file located in the back of the ambulance. A more detailed description of the use of guidelines in the organization can be found in a recent study [3].

The intervention period was March, April and May 2013. February was not included, since training on the CDSS was conducted on three occasions that month. The training consisted of a lecture in which the CDSS functions were presented, followed by manikin training with the CDSS, for a total of four hours. Apart from the training on the CDSS, no other events which could have influenced the outcomes were identified.

The main outcome was compliance with the basic assessment of the medical patients described in the local and national prehospital guidelines [26,27], which are in turn guided by the content of the Advanced Medical Life Support (AMLS) system [23]. The second outcome was OST, defined as the time when the ambulance arrives at the address to the time when transportation is initiated. The two outcomes were compiled weekly during the study period. The objective of assessing the outcome weekly is dependent on the fact that, during the course of a week, six to eight of the 10 participants were on duty and expected individual differences should even themselves out during a week. As the study was ongoing for six months and there was one data point every week, the study had 24 data points.

Since power calculation is difficult in time series, no power was calculated. Instead a power calculation from a simulation study [28] was used. The simulation-based power calculation displayed that with 12 data points in the pre-intervention period and 12 data points in the post-intervention period, there is more than $80 \%$ chance to obtain an effect size of 1.0 or more. The power is increased when the effect size is increased. The power are also dependent of the degree of auto correlation there data with a low degree of auto correlation have a higher power [28].

A rating protocol was developed for the study (see Additional file 1). The protocol was based on the description of how to assess patients with medical emergencies in the local and national guidelines [26,27]. The protocol consists of 33 assessment interventions and a protocol in which all 33 interventions were completed was defined as $100 \%$ compliance. Two of the authors, (MAH, BOS) was pilot-tested the rating protocol. They separately rated the same 10 records and their interrater reliability (IRR) was calculated using Cohen's $k$. The IRR was found to be = kappa $0.75(\mathrm{p}=0.001)$ which is considered as a substantial agreement [29].

Eligible patients for the study were searched for in the ambulance organization's patient record database, according to directions for inclusion and exclusion criteria. Patient records from included patients were extracted by one of the authors (MAH). We estimate that the extraction process identified 90 to $100 \%$ of the eligible patients.

The final rating was performed by one of the authors (MAH) who was reading the eligible ambulance patient records and identified information which describes assessment interventions included in the rating protocol. Interventions not described in the patients' records were regarded as not having been performed. Since the primary variables were not entirely objective, the rater was blinded to the period to which the record belonged. Two co-authors (AJ, BOS) removed the date of the included records and, using a coding process, the rated records could be put in the right time period prior to analysis. The records were also randomized in time periods, as we expected the rating to change with time.

The information in the CDSS was not collected, since we wanted the same data sources in the baseline period and the intervention period. The participants in the study were aware of data collection in both the pre- and post-intervention phases. 


\section{Analysis}

For demographic data, descriptive statistics were used. To determine the distribution of eligible patients between the pre- and post-intervention phase, an independent T-test was used for continuous data and chi-square tests for categorical data.

To analyze the difference between the pre- and postintervention phase, the regression was tested for autocorrelation in the residuals using the Durbin-Watson test.

A segmented regression analysis was performed to determine the level and slope in the pre-intervention phase and the change in level and slope in the post-intervention phase [24] on the mean percentage compliance and mean OST. The full regression model included changes in slope in the pre- and post-intervention phase and changes in level after the introduction of the CDSS. For the most parsimonious model, non-significant variables were removed stepwise for entry in the model with P-in 0.05 and P-out 0.10 .

A p-value of $<0.05$ was considered significant in all statistical tests.

All analyses were performed using SPSS 21.0 (SPSS, Inc, Chicago, IL).

\section{Ethical issues}

One ethical issue when it comes to the collected design is that a limited number of individuals are closely evaluated. There is a risk that the participants may feel criticized in their professional role. The participants were guaranteed anonymity and were told that all study data would be treated confidentially. Informed written consent was obtained from all the participants in the study and they were informed that they were free to withdraw from the study at any time and that the study data relating to that participant would be erased.

The study was approved by the Regional Ethics Committee, Gothenburg, Sweden (Dnr: 1133-11).

\section{Result}

A total of 371 patients were included in the study. There was a difference in the distribution between the pre- and post- intervention phases according to gender and age. The patients in the post-intervention phase were two years older on average compared with those in the preintervention phase $(p=0.04)$ and there were significantly more male patients in the post-intervention phase $(p=0.04)$. There was no significant difference in the distribution of the diagnostic categories between the phases $(p=0.86)$. There was also no significant difference in the distribution of eligible patients per participating member of prehospital clinicians in the pre- and post-intervention period $(p=0.29)$ (Table 1$)$.
The single data points (week) comprised the ratings of 15.52 (mean) medical records, with a range of 9 to 29 records. The test for autocorrelation revealed that no autocorrelation was present. The Durbin-Watson was found to be 2.118 when the dependent variable is "mean percentage of compliance" and 2.074 with "OST" as the dependent variable. The level for no autocorrelation is 1.55-2.45.

Just before the baseline period, the prehospital clinicians compliance with the prehospital guidelines for assessment was an average of $53 \%(p=<0.001)$. There was no significant change in slope in the pre-intervention phase $(p=0.639)$. After the introduction of the CDSS, there was a significant jump in the level of compliance with the assessment process. The compliance rose by $10 \%$ $(p=<0.001)$. The post-intervention slope was also stable, with a non-significant change $(p=0.912)$. The most parsimonious model contained only the intercept and the change in level after non-significant parameters was removed stepwise (Table 2, Figure 1).

The introduction of the CDSS had no significant effect on the time spent on scene. The most parsimonious model contained the intercept $(p=<0.001)$ and change in pre-intervention slope $(p=<0.001)$. The result indicates that the OST had already increased before the introduction of the CDSS (Table 3, Figure 2). The reason for the positive pre-intervention slope is unknown.

\section{Discussion}

The result of the present study indicates that the use of the CDSS can increase compliance with the basic assessment process described in the prehospital guidelines. The clinical study confirmed the result of a previous simulation study of the same CDSS. In the earlier simulation study the use of the CDSS increased the compliance to guidelines in two simulated patient cases from $60 \%$ compliance in the control group to $80 \%$ in the CDSS group. The greatest difference in compliance was in the first assessment (ABCD) and in the anamnesis of the patient [30]. The greatest advantage of the CDSS in the prehospital setting is probably the format. In a case study of the use of guidelines and protocols in a prehospital organization, the format of the guidelines was the main obstacle to using the guidelines. In general, the prehospital clinicians were in favor of using the guidelines and they regarded the guidelines as an absolute necessity, but the paper format makes the guidelines difficult to use in patient assessments [3]. The visibility and usability of the guidelines have also been identified as important features for guideline use in other settings [31].

As an effect of improved compliance with the guidelines, the prehospital clinicians performed more assessments and interventions when using the CDSS compared with the pre-intervention phase. How can these results 
Table 2 Results of segmented regression analysis of the impact of CDSS of compliance to assessment process described in prehospital guidelines

\begin{tabular}{|c|c|c|c|c|}
\hline & Coefficient & Standard error & $t$-statistic & $P$-value \\
\hline \multicolumn{5}{|l|}{ Full regression model } \\
\hline Intercept & 53.194 & 1.469 & 36.201 & $<0.001$ \\
\hline Slope before CDSS & -0.097 & 0.200 & -0.483 & 0.634 \\
\hline Change in level after CDSS & 10.637 & 1.960 & 5.428 & $<0.001$ \\
\hline Change in slope after CDSS & 0.031 & 0.282 & 0.111 & 0.912 \\
\hline \multicolumn{5}{|c|}{ The most parsimonious model } \\
\hline Intercept & 52.567 & 0.663 & 79.324 & $<0.001$ \\
\hline Change in level after CDSS & 9.683 & 0.937 & 10.332 & $<0.001$ \\
\hline
\end{tabular}

have an effect on patient safety issues in the prehospital setting? The single most common type of human error is leaving out necessary task steps [32]. There are several reasons for the omissions; informational overload, the procedural steps are not entirely logical, premature exits in which procedures at the end of a task are left out and unexpected interruptions in the task process [32]. In a previous study, the ability to collect and use information was one of the greatest differences between expert paramedics and novices. The expert paramedics also performed more interventions and assessments compared with the less experienced paramedics [33]. An accurate prehospital assessments have been found to benefit patients with stroke [34] and ST-elevation myocardial infarctions [35]. The early prehospital identification of the symptoms shortens the time to definite care and consequently reduces mortality and complications. The ability to collect useful information and organize the information in order to sort useful information from irrelevant information is also a characteristic of the expert nurse [36]. Experienced clinicians often use schedules based on previous experience to organize information collection [36]. The CDSS in the present study can be defined as a schedule of this kind and could have the potential to reduce cognitive overload and avoid "short cuts" in the decision making process. Good clinical information systems can have a positive effect on clinical reasoning. Physicians who start using an Electronic Health Record (EHR) change the way they make decisions compared with using paper-based health records. After using the system, the physicians were more focused on problem solving with simple propositions. The cognitive changes were sustained even after the study [37]. The reason for the cognitive change is probably the way the information is processed, stored and presented [36].

However, information systems can also have negative consequences. Patel et al. [38] argue that CDSS mediate human performance. There is a risk, for example that the CDSS will slow down the development of changes in knowledge and skills [38]. There is differences in how expert and novice clinicians use CDSS. Studies have shown [37] that the experienced decision maker (expert) uses the CDSS structure and is able to complete the task

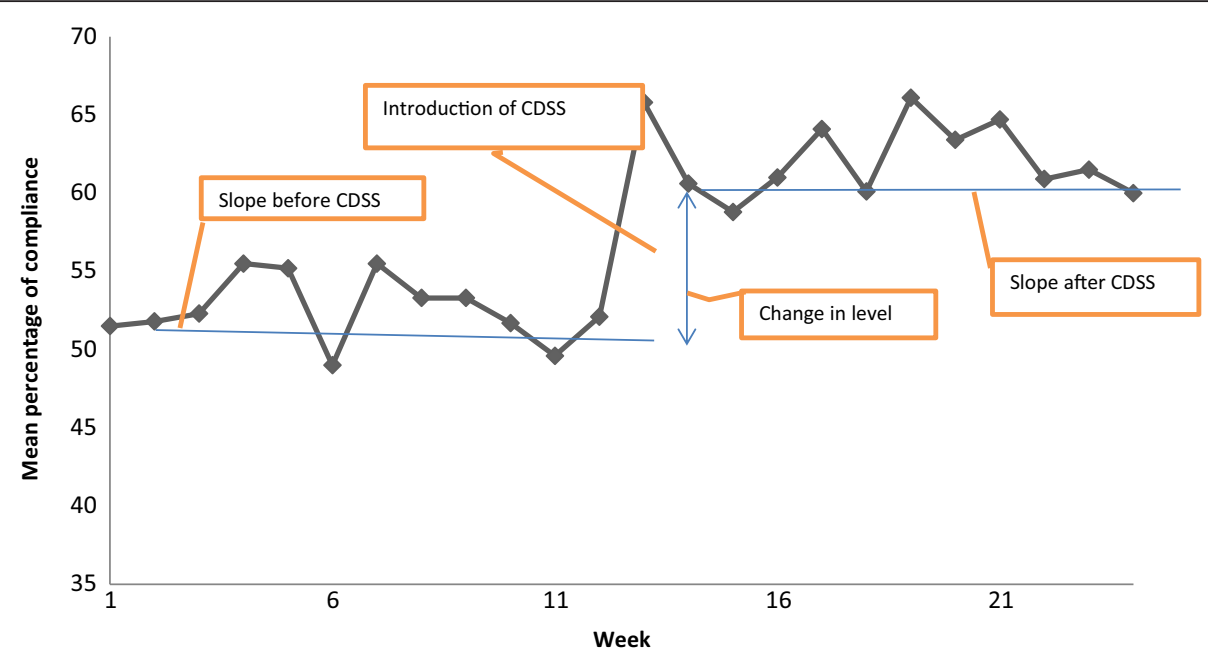

Figure 1 Mean percentage compliance of prehospital guidelines of the assessment process. 
Table 3 Results of segmented regression analysis of the CDSS impact of On Scene Time (OST)

\begin{tabular}{|c|c|c|c|c|}
\hline & Coefficient & Standard error & $t$-statistic & $P$-value \\
\hline \multicolumn{5}{|l|}{ Full regression model } \\
\hline Intercept & 11.712 & 1.001 & 11.703 & $<0.001$ \\
\hline Slope before CDSS & 0.185 & 0.136 & 1.363 & 0.188 \\
\hline Change in level after CDSS & 0.670 & 1.335 & 0.502 & 0.621 \\
\hline Change in slope after CDSS & -0.073 & 0.192 & -0.382 & 0.702 \\
\hline \multicolumn{5}{|c|}{ The most parsimonious model } \\
\hline Intercept & 11.772 & 0.659 & 17.855 & $<0.001$ \\
\hline Slope before CDSS & 0.188 & 0.046 & 4.080 & $<0.001$ \\
\hline
\end{tabular}

using the structure, while a novice uses the CDSS to prompt and gather too much irrelevant information, which results in incorrect decisions. The expert appears to use guidelines and algorithms as a problem-solving process, whereas the novice uses the same system as an educational device $[37,38]$.

The CDSS in the present study had no significant effect on OST, in spite of the fact that more assessments and interventions were performed. This result is in line with a previous study which reveals no relationship between the number of performed interventions and OST [39]. It is possible that, by organizing the assessment process in a CDSS, the ambulance team can work in a more organized way on the scene and as a result produce more in a shorter time.

The CDSS level of usability is also an important feature, especially in the prehospital setting, which can be described as unstable. Since we were not able to configure the CDSS with the ordinary prehospital records system, the prehospital clinicians had to document patient information twice, first in the CDSS and later in the ordinary ambulance patient record system in a computer at the ED.
This double documentation probably has limited effect of the OST, but potentially increase the total prehospital time, which was not measured in this study.

The present study has several limitations. Firstly, it is important to remember that the present results relate to this CDSS in this particular context. A CDSS is a complex intervention [40]. The complexity of an intervention is determined by two variables, the number of components and the level of interrelatedness [41]. In these terms, a CDSS used in a prehospital setting can be defined as having a medium level of complexity. There are relatively few components (the ambulance team and the patient), but the components have a high degree of interrelatedness (e.g. computer interface, usability, structure, user compliance, acceptance, work culture and so on.) The results of a complex intervention study can be difficult to transfer to other settings. To increase the transferability, a deep context description is important [42]. Secondly, the study was not randomized. The ITS design is a way of strengthening the "before and after study" by controlling for secular trends, cyclical effects and random fluctuations [25]. The use of the "Quality Criteria for Interrupted Time Series

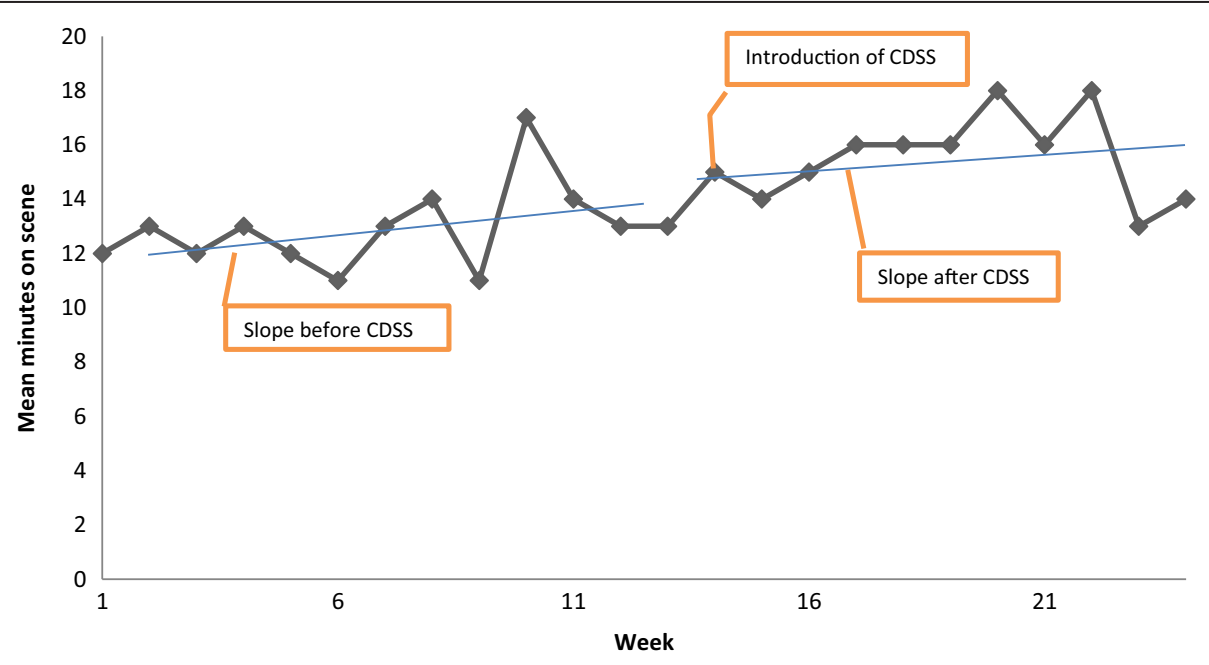

Figure 2 Mean On Scene Time (OST). 
Designs" [25] reveal the importance of blinding the rater when it is possible. In the present study it was handle by a process were co-authors were removed the date on the included records so the rater did not know if they belong to the baseline or intervention period. Another important issue is the quality of the protocols used in the measurements. One way to strengthen the rating protocol reliability is to determine the IRR score. In the present study the IRR was calculated to kappa $0.75(p=0.001)$, which is considered as a substantial agreement [29]. Thirdly, the primary variable is based on data extracted from Electronic Health Records (EHR). There is a substantial risk of data loss and the information in the EHR may not entirely reflect reality [43]. Fourthly, the study did not investigate the relationship between the increase in guideline compliance and patient outcomes. Future studies should concentrate on the way increased guideline compliance in the prehospital setting can affect outcomes such as mortality, morbidity, time in hospital, complications and time to definite care.

\section{Conclusions}

In this interrupted time-series study, the use of the CDSS increased compliance with the basic assessment process of a patient with a medical emergency described in the local and national prehospital guidelines. One effect of the increased compliance was that the ambulance staff performed more prehospital assessments and interventions, which should help to increase patient safety. There was no significant change in the time spent on scene when the CDSS was used.

\section{Additional file}

Additional file 1: Assessment elements of the medical patient $(n=33)$.

\section{Competing interests}

The authors declare that they have no competing interests.

\section{Authors' contributions}

MAH was designing the study, participated in the data collection, participated in the analyses of data and drafted the manuscript. BOS and AJ were participated in the design process and helped to draft the manuscript. BAS made substantial contribution to the development of the CDSS and helped to draft the manuscript. BAG and $\mathrm{MH}$ were participated in the drafting of the manuscript. All authors read and approved the final manuscript.

\section{Acknowledgement}

We would like to thank the ambulance nurses and EMTs at the participated ambulance organization. We would also like to thank Alexis Palma, University of Borås and Mats Nilsson, Futurum in Jönköping for statistical support.

\section{Author details}

${ }^{1}$ University of Borås, School of Health Sciences, 50190 Borås, Sweden.

${ }^{2}$ School of Health Sciences, Jönköping University, Jönköping 551 11, Sweden.

${ }^{3}$ Department of Signals and Systems, Chalmers University of Technology,

Gothenburg, Sweden.
Received: 1 October 2013 Accepted: 6 August 2014

Published: 9 August 2014

\section{References}

1. Jensen $\mathrm{J}$ : Paramedic clinical decision-making: result of two Canadian studies. J Paramed Pract 2011, 1:63-71.

2. Jensen $J$, Croskerry P, Travers A, EMS: Consensus on paramedic clinical decisions during high-acuity emergency calls: results of a Canadian Delphi study. J Can Assoc Emerg Phys 2011, 13(5):310-318.

3. Hagiwara M, Suserud B-O, Jonsson A, Henricson M: Exclusion of context knowledge in the development of prehospital guidelines: results produced by realistic evaluation. Scand J Trauma Resusc Emerg Med 2013 21(1):46.

4. Bigham BL, Bull E, Morrison M, Burgess R, Maher J, Brooks SC, Morrison LJ: Patient safety in emergency medical services: executive summary and recommendations from the Niagara Summit. Can J Emerg Med Care 2011, 13(1):13-18.

5. Atack L, Maher J: Emergency medical and health providers' perceptions of key issues in prehospital patient safety. Prehosp Emerg Care 2010, 14(1):95-102.

6. Brice JH, Studnek JR, Bigham BL, Martin-Gill C, Custalow CB, Hawkins E, Morrison $L$ : EMS provider and patient safety during response and transport: proceedings of an ambulance safety conference. Prehosp Emerg Care 2012, 16(1):3-19.

7. Patterson PD, Weaver MD, Frank RC, Warner CW, Martin-Gill C, Guyette FX, Hubble MW, Songer TJ, Callaway CW, Kelsey SF, Hostler D: Association between poor sleep, fatigue, and safety outcomes in emergency medical services providers. Prehosp Emerg Care 2012, 16(1):86-97.

8. Patterson PD, Huang DT, Fairbanks RJ, Simeone S, Weaver M, Wang HE: Variation in emergency medical services workplace safety culture. Prehosp Emerg Care 2010, 14(4):448-460.

9. Cone DC: Knowledge translation in the emergency medical services: a research agenda for advancing prehospital care. Acad Emerg Med 2007, 14(11):1052-1057.

10. Taira BR, Singer AJ, Cassara G, Salama MN, Sandoval S: Rates of compliance with first aid recommendations in burn patients. J Burn Care Res 2010, 31(1):121-124

11. Bosse G, Schmidbauer W, Spies CD, Sörensen M, Francis RCE, Bubser F, Krebs M, Kerner T: Adherence to guideline-based standard operating procedures in pre-hospital emergency patients with chronic obstructive pulmonary disease. J Int Med Res 2011, 39(1):267-276.

12. Fisher JD, Vinci RJ: Prehospital management of pediatric asthma requiring hospitalization. Pediatr Emerg Care 1995, 11(4):217-219.

13. Rittenberger JC, Beck PW, Paris PM: Errors of omission in the treatment of prehospital chest pain patients. Prehosp Emerg Care 2005, 9(1):2-7.

14. Woollard M, Smith A, Elwood P: Pre-hospital aspirin for suspected myocardial infarction and acute coronary syndromes: a headache for paramedics? Emerg Med J 2001, 18(6):478-481.

15. Kinsman L, Tori K, Endacott R, Sharp M: Guideline implementation fails to improve thrombolytic administration. Accid Emerg Nurs 2007, 15(1):27-33.

16. Rycroft-Malone J, Fontenla M, Bick D, Seers K: A realistic evaluation: the case of protocolbased care. Implement Sci 2010, 5:38-48.

17. Sahota N, Lloyd R, Ramakrishna A, Mackay JA, Prorok JC, Weise-Kelly L, Navarro T, Wilczynski NL, Haynes RB: Computerized clinical decision support systems for acute care management: a decision-maker-researcher partnership systematic review of effects on process of care and patient outcomes. Implement Sci 2011, 6:91

18. Kawamoto K, Houlihan CA, Balas EA, Lobach DF: Improving clinical practice using clinical decision support systems: a systematic review of trials to identify features critical to success. Br Med J 2005, 330(7494):765-768.

19. Hagiwara M, Henricson M, Jonsson A, Suserud B-O: Decision-support tool in prehospital care: a systematic review of randomized trials. Prehosp Disaster Med 2011, 26(5):319-329.

20. Huckvale C, Car J, Akiyama M, Jaafar S, Khoja T, Bin Khalid A, Sheikh A, Majeed A: Information technology for patient safety. Qual Saf Health Care 2010, 19(Suppl 2):25-33.

21. Gonzalez RP, Cummings GR, Phelan HA, Mulekar MS, Rodning CB: Does increased emergency medical services prehospital time affect patient mortality in rural motor vehicle crashes? A statewide analysis. Am J Surg 2009, 197(1):30-34. 
22. Greenes RA: Clinical decision support: the road ahead. Amsterdam: Elsevier Academic Press; 2007.

23. Dalton AL: Advanced Medical Life Support: a Practical Approach to Adult Medical Emergencies. Upper Saddle River, N.J: Pearson; 2010.

24. Wagner AK, Soumerai SB, Zhang F, Ross-Degnan D: Segmented regression analysis of interrupted time series studies in medication use research. J Clin Pharm Ther 2002, 27(4):299-309.

25. Ramsay CR, Matowe L, Grilli R, Grimshaw JM, Thomas RE: Interrupted time series designs in health technology assessment: lessons from two systematic reviews of behavior change strategies. Int J Technol Assess Health Care 2003, 19(4):613-623.

26. Södra Älvsborgs Sjukhus: Behandlingsanvisningar (Treatment instructions). Available at: http://sas.vgregion.se/upload/S\%C3\%84S/Ambulanssjukv\%C3\% A5rd/Behandlingsriktlinjer2011-05-01.pdf. Accessed January 26, 2012.

27. SLAS: Behandlingriktlinjer (Treatment guidelines). Available at: http:// www.flisa.nu/web/page.aspx?refid=18. Accessed January 26, 2012.

28. Zhang F, Wagner AK, Ross-Degnan D: Simulation-based power calculation for designing interrupted time series analyses of health policy interventions. J Clin Epidemiol 2011, 64(11):1252-1261.

29. Viera AJGJ: Understanding Interobserver Agreement: The Kappa Statistic. Fam Med 2005, 37(5):360-363.

30. Hagiwara MA, Sjöqvist BA, Lundberg L, Suserud B-O, Henricson M, Jonsson $A$ : Decision support system in prehospital care: a randomized controlled simulation study. Am J Emerg Med 2013, 31(1):145-153.

31. Rycroft-Malone JFM, Bick D, Seers K: Protocol-based care evaluation project. Final report for the national institute for health research service delivery and organisation programme 2010. Retievered from: http://www. sdo.nihr.ac.uk/projdetails.php?ref=08-1405-078.

32. Reason J: Combating omission errors through task analysis and good reminders. Qual Saf Health Care 2002, 11(1):40-44.

33. Smith MW, Bentley MA, Fernandez AR, Gibson G, Schweikhart SB, Woods DD: Performance of experienced versus less experienced paramedics in managing challenging scenarios: a cognitive task analysis study. Ann Emerg Med 2013, 17(13):00416-00512.

34. Rajajee V, Saver J: Prehospital care of the acute stroke patient. Tech Vasc Interv Radiol 2005, 8(2):74-80

35. Bång A, Grip L, Herlitz J, Kihlgren S, Karlsson T, Caidahl K, Hartford M: Lower mortality after prehospital recognition and treatment followed by fast tracking to coronary care compared with admittance via emergency department in patients with ST-elevation myocardial infarction. Int $J$ Cardiol 2008, 129(3):325-332.

36. Patel VL, Currie LM: Clinical cognition and biomedical informatics: issues of patient safety. Int J Med Inform 2005, 74(11-12):869-885.

37. Patel VL, Kushniruk AW, Yang S, Yale J-F: Impact of a computer-based patient record system on data collection, knowledge organization, and reasoning. J Am Med Inform Assoc 2000, 7(6):569-585.

38. Patel VL, Kaufman DR, Arocha JF: Emerging paradigms of cognition in medical decision-making. J Biomed Inform 2002, 35(1):52-75.

39. van der Velden MWA, Ringburg AN, Bergs EA, Steyerberg EW, Patka P, Schipper IB: Prehospital interventions: time wasted or time saved? An observational cohort study of management in initial trauma care. Emerg Med J 2008, 25(7):444-449.

40. Campbell M, Fitzpatrick R, Haines A, Kinmonth AL, Sandercock P, Spiegelhalter D, Tyrer D: Framework for design and evaluation of complex interventions to improve health. BMJ 2000, 321(7262):694-696.

41. Kannampallil TG, Schauer GF, Cohen T, Patel VL: Considering complexity in healthcare systems. J Biomed Inform 2011, 44(6):943-947.

42. Øvretveit J: Understanding the conditions for improvement: research to discover which context influences affect improvement success. BMJ Qual Saf 2011, 20(Suppl 1):18-23.

43. Chan KS, Fowles JB, Weiner JP: Review: electronic health records and the reliability and validity of quality measures: a review of the literature. Med Care Res Rev 2010, 67(5):503-527.

doi:10.1186/1472-6947-14-70

Cite this article as: Andersson Hagiwara et al: : The effect of a

Computerised Decision Support System (CDSS) on compliance with the prehospital assessment process: results of an interrupted time-series study. BMC Medical Informatics and Decision Making 2014 14:70.

\section{Submit your next manuscript to BioMed Central and take full advantage of:}

- Convenient online submission

- Thorough peer review

- No space constraints or color figure charges

- Immediate publication on acceptance

- Inclusion in PubMed, CAS, Scopus and Google Scholar

- Research which is freely available for redistribution 